\title{
Impact of COVID-19 on incidence and treatment of intracranial aneurysms in Poland: a national study
}

\author{
Grzegorz Miękisiak $^{1^{*}}$, Justyna Fercho ${ }^{2^{*}}$, Samuel D. Pettersson ${ }^{3^{*}}$, Tomasz Szmuda ${ }^{2^{*}}$, Paweł Słoniewski ${ }^{2}$ \\ ${ }^{*}$ Contributed equally to the manuscript \\ ${ }^{1}$ Institute of Medicine, Opole University, Poland \\ ${ }^{2}$ Neurosurgery Department, Medical University of Gdansk, Poland \\ ${ }^{3}$ Scientific Circle of Neurology and Neurosurgery, Neurosurgery Department, \\ Medical University of Gdansk, Poland
}

\begin{abstract}
Introduction. The COVID-19 pandemic greatly disrupted the national healthcare system in Poland, resulting in the implementation of new protocols allowing only patients with severe diagnoses to receive immediate treatment. Given that an intracranial aneurysm (IA) is regarded as one of the most severe diagnoses, we planned to assess whether the current protocol has successfully provided the standard treatment for IAs.

Material and methods. Data on all IA cases treated from 2015 to 2020 was extracted from the JGP (a homogeneous group of patients) catalogue provided by the National Health Index of Poland (NFZ, Narodowy Fundusz Zdrowia). Poisson regression was used to determine the significance of the change in hospital admissions, and differences between proportions were analysed using the " $\mathrm{N}-1$ " $\mathrm{Ch}$-squared test.

Results. A total of 21,801 IA patients treated during 2015-2020 were included in this study. The overall number of hospitalisations due to IAs fell in the open surgery group, but not in the endovascular cohort. Mortality rates following IA treatment increased significantly by $21 \%$ in 2020 compared to preceding years. The demographics changed as well; the patients were significantly younger during the pandemic.

Conclusions. Our findings show that the current strategy for optimal care for patients diagnosed with IAs in Poland during the pandemic is failing to maintain high quality treatment. New methods to improve the current plan should be implemented to address future crises.
\end{abstract}

Key words: COVID-19, intracranial aneurysm, mortality, incidence

(Neurol Neurochir Pol 2022; 56 (1): 96-100)

\section{Introduction}

The COVID-19 pandemic continues to disrupt healthcare globally to an unprecedented scale. It has had a profound impact on the management of neurosurgical patients: a recent global study identified that neurosurgical operations have more than halved since the start of the pandemic [1]. As the healthcare system became overwhelmed by the pandemic, all other health issues were 'brushed aside' and the majority of non-emergency procedures were deferred [2].
In this study, we focused on patients diagnosed with intracranial aneurysms (IAs), one of the most severe neurological conditions wherein, when ruptured, sudden death is reported to occur in $10-15 \%$ of individuals, and $50 \%$ of survivors suffer from permanent disabilities [3]. By evaluating the national report on patients diagnosed with IAs during the past five years, this study identifies whether the ongoing pandemic is preventing patients from receiving the required treatment for IAs in Poland.

Address for correspondence: Justyna Fercho, Medical University of Gdansk, Poland; e-mail: jfercho@uck.gda.pl 


\section{Material and methods}

Data on all IA cases from 2015 to 2020 was extracted from the National Health Index of Poland (NFZ, Narodowy Fundusz Zdrowia) presenting a set of data in the area of healthcare statistics in the general, billing and medical dimensions, relating to products from catalogues such as: the JGP (a homogeneous group of patients), the Separate Benefits, the Benefits to be Summed, the Radiation Therapy Services and the Highly Specialised Services. In this study, data was extracted from the JGP catalogue for section A (nervous system diseases) under group A11 (comprehensive intracranial procedures), section Q (vascular diseases) under groups Q31(Haemorrhagic disorders of cerebral vessels-vascular remodelling with the use of a stent), Q32 (Haemorrhagic disorders of cerebral vessels-large endovascular repair procedure), and Q33 (Haemorrhagic disorders of cerebral vessels-medium endovascular repair procedure). For each group (A11, Q31-Q33) general data was selected: sex of patient, age of patient and mode of discharge, as well as medical data: ICD 9 and ICD10 procedures for treatment of intracranial aneurysms.

Poisson regression was used to determine the significance of the change in hospital admissions. The differences between proportions were analysed using the "N-1" Chi-squared test. Statistical analyses were carried out using StatsDirect version 3.3.5 (StatsDirect Ltd, Merseyside, UK; http:.//www.statsdirect. com) and MedCalc v. 12.5.0.0 (MedCalc Software, Ostend, Belgium).

\section{Results}

In 2015-2020, a total of 21,801 hospitalisations due to IAs were recorded. We observed a decrease in hospitalisations for IAs in 2020 compared to 2015-2019. This was a consequence of a drop in the number of open surgery cases, and the difference was statistically significant at $\mathrm{p}<0.05$. We noticed some changes in the demographics. The population of patients was significantly younger in 2020, and there were relatively more males. Most importantly, the mortality rate for patients diagnosed with AIs in 2020 was significantly higher than in the preceding five years, with an increase of approximately $21 \%$ compared to the mean of the previous years. The data is set out in Table 1.

\section{Discussion}

Poland has suffered from COVID-19 as have all countries in the European Union. The first case of SARS-CoV-2 was registered on 4 March, 2020. Soon after, restrictions were introduced. On 12 March, a national state of emergency was declared in Poland, and three days later the borders were sealed. All citizens were required to stay at home unless absolutely necessary, and on 20 March the prime minister announced an epidemic state in Poland. The initial lockdown proved effective, and therefore a gradual lifting of the restrictions began on 20 April. In October, a second COVID-19 wave hit the country, resulting in a peak number of cases much higher than the first one. Restrictions were brought in again and remained in effect until the end of the year. According to official sources, in 2020 there were 1,294,878 cases of SARS-CoV-2, with 28,554 confirmed deaths, nationwide [4]. The national healthcare system was grossly overburdened from mid-March throughout the remainder of the year.

As the country was seriously affected during this time, so in particular was the healthcare system. Almost all hospitals were required to implement a protocol forcing only those patients with life-threatening emergencies to be treated without delay. An IA haemorrhage clearly falls into this category.

\section{Treatment}

In general, treatment methods for IAs come in two forms: open surgery and endovascular. The open surgical intervention involves 'clipping' whereby a titanium clip is attached across the neck to obliterate the malformation. The endovascular intervention is a less invasive method wherein a micro-catheter is inserted, typically through the femoral artery, and reaches the IA where it detaches a coil in the lumen of the aneurysm to stabilise the region. Both intervention types come with their own complications however, and an up-to-date Cochrane review has identified the endovascular method as the safer intervention for IAs [5], especially for unruptured IAs [6].

Our study showed that open surgical treatment for IAs decreased in 2020 compared to the mean of 2015-2019 $(\mathrm{p}<0.05)$, while the number of endovascular procedures did not change. This was probably caused by the limited availability of neurosurgical units as many were turned into 'COVID-only' dedicated departments. On the other hand, endovascular treatment in Poland is highly centralised and was largely unaffected by the pandemic. Interestingly, the number of non-urgent unruptured aneurysms treated did not change as one would expect. The incidence of aneurysmatic subarachnoid haemorrhage (aSAH) in Poland during 2020 did not change significantly, and similar results were published in a Scandinavian study [7]. However, in one pan-European study [8], as many as $80 \%$ of neurosurgical departments required rationing, and a significant decline in the ratio of ICU-treated SAH patients was noted. Testimony to the dire situation in which European neurosurgery found itself comes from an article published on behalf of the European Association of Neurosurgical Societies in May 2020 [9] concerning the ethical issues related to the triage of neurosurgical patients. This report provided a valuable framework for decision making during difficult times with limited availability of resources.

As the pandemic swept around the world, several survival strategies were proposed by authors. Lombardy in Italy was one of the first regions in the world to be heavily affected by the outbreak. Neurosurgeons practicing in this area swiftly adapted to the situation by introducing a 'Hub and Spoke' system which 


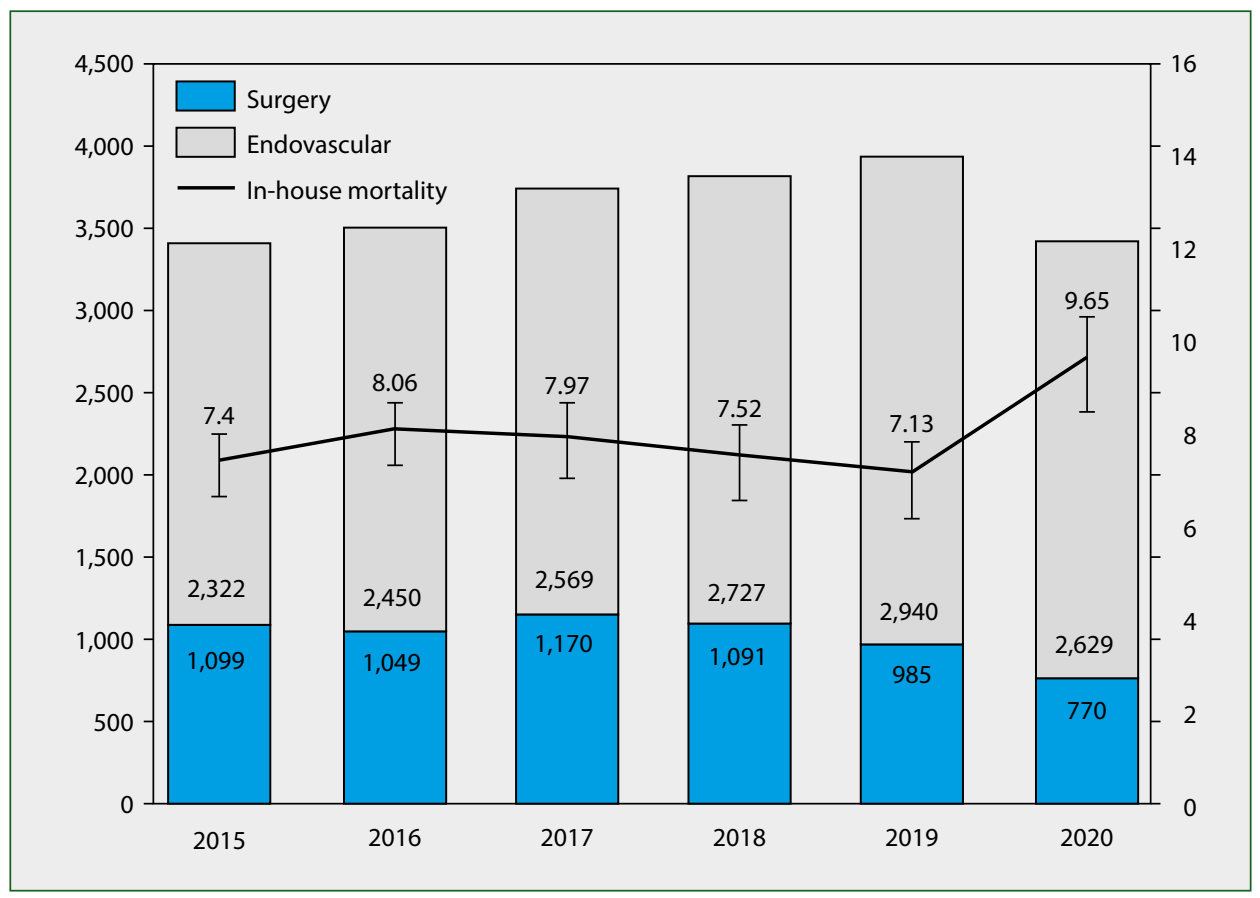

Figure 1. Comparison of annual intracranial aneurysm patient volume between pre-COVID-19 period and 2020

avoided therapeutic delay and led to results overall that were comparable to the control period [10]. The EANS Individual Membership Committee emphasised that neurosurgeons should learn a somewhat new skill of reactive management [11]. They were encouraged to share their experiences at the transnational level and act as a team, rather than individuals. Indeed, this idea prompted us to write this very article.

\section{Demographics}

The age groups of patients diagnosed with IAs significantly differed during 2020. An overall increase in younger patients was observed during the pandemic versus older patients (aged 61-80) where diagnoses decreased significantly. It was identified early in the pandemic that the COVID-19 infection is much more hazardous in the elderly population [12]. Given that most unruptured IAs are identified incidentally during neuroimaging for other reasons, due to their insidious onset [13], this change in age groups could be the result of older patients deferring hospital treatment of unruptured aneurysms for the above reasons.

\section{In-hospital mortality rate}

Our findings identified a highly significant increase in the in-hospital mortality rates of patients treated for IAs compared to the previous years. With 2015-2019 averaging a 7.61\% mortality rate, this increased by $21 \%$ in 2020 , resulting in an annual mortality rate of $9.65 \%$. The baseline figure is similar to the earlier study by Tykocki et al. [14]. This increase is a result of multiple factors, but the limited availability of intensive care unit (ICU) beds was probably the most important one.
The increase was noticed in all treatment groups, and thus the change of treatment paradigm in favour of endovascular techniques did not play a role. One would expect that the treatment of non-emergency unruptured aneurysms would be postponed during a pandemic, and that thus more poor-grade patients would result in worse outcomes. However, this was not the case, as the overall number of treated unruptured aneurysms did not change. COVID-19 infection as a comorbidity probably played some role in the overall outcome [15], but this was likely to be negligible as the probability of coincidence was very low.

\section{Limitations}

Patients with mild symptoms of haemorrhage, typically a slight headache, are less likely to seek medical attention when this is of limited availability. Therefore, the actual incidence of non-lethal haemorrhages during a pandemic may differ to a certain extent.

\section{Conclusions}

The COVID-19 pandemic has seriously affected Poland as a whole, including its healthcare system. The system was transitioned at very short notice to deal with the burden of the pandemic, meaning that other health issues were unavoidably sidelined. This caused a shift of the treatment paradigm of AIs and most importantly it negatively affected the quality of care as the mortality rate spiked in 2020. Overall, no significant change in the incidence of ruptured IAs was noted. Our study implies that the management of neurosurgical emergencies, such as aSAH, relies heavily on access to intensive care units. 
Table 1. Comparison of intracranial aneurysm patient demographics, procedures, diagnoses, and mortality rate between pre-COVID period and 2020

\begin{tabular}{|c|c|c|c|c|c|}
\hline \multirow{2}{*}{ General Statistics } & \multicolumn{2}{|c|}{2020} & \multicolumn{2}{|c|}{$2014-2019$} & \multirow[t]{2}{*}{$\begin{array}{l}\text { Statistical } \\
\text { significance }\end{array}$} \\
\hline & & & & & \\
\hline Total hospitalisations [n (per annum)] & \multicolumn{2}{|l|}{3,399} & \multicolumn{2}{|c|}{$18,402(3,680)$} & \\
\hline Open surgery [n (per annum)] & \multicolumn{2}{|l|}{770} & \multicolumn{2}{|c|}{$5,394(1,078)$} & $p<0.05^{* *}$ \\
\hline Endovascular treatment [n (per annum)] & \multicolumn{2}{|l|}{2,629} & \multicolumn{2}{|c|}{$13,008(2,601)$} & \\
\hline$\%$ clipped & \multicolumn{2}{|l|}{22.65} & \multicolumn{2}{|l|}{29.31} & \\
\hline \multicolumn{6}{|l|}{ Demographics: sex gender } \\
\hline F:M ratio & \multicolumn{2}{|l|}{1.98} & \multicolumn{2}{|l|}{2.15} & \\
\hline \multicolumn{6}{|l|}{ Demographics: age } \\
\hline Age group & $\mathrm{n}$ & $\%$ & $\mathrm{n}$ & $\%$ & \\
\hline$<1$ & 2 & 0.08 & 33 & 0.18 & \\
\hline $1-6$ & 23 & 0.68 & 60 & 0.33 & $\mathrm{p}<0.01^{*}$ \\
\hline $7-17$ & 161 & 4.74 & 296 & 1.61 & $\mathrm{p}<0.0001^{*}$ \\
\hline $18-40$ & 727 & 21.39 & 2,949 & 16.03 & $\mathrm{p}<0.0001^{*}$ \\
\hline $41-60$ & 1,378 & 40.56 & 7,620 & 41.41 & \\
\hline $61-80$ & 1,008 & 29.66 & 6,955 & 37.8 & $p<0.0001^{*}$ \\
\hline 81 and more & 97 & 2.88 & 485 & 2.64 & \\
\hline \multicolumn{6}{|l|}{ Ruptured vs. unruptured } \\
\hline Unruptured aneurysm [n (per annum)] & \multicolumn{2}{|l|}{1,057} & \multicolumn{2}{|c|}{$5,667(1133)$} & \\
\hline Ruptured aneurysm [n (per annum)] & \multicolumn{2}{|l|}{2,339} & \multicolumn{2}{|c|}{$12,731(2,546)$} & \\
\hline$\%$ unruptured & \multicolumn{2}{|l|}{31.12} & \multicolumn{2}{|l|}{30.8} & \\
\hline \multicolumn{6}{|l|}{ Procedures performed } \\
\hline Stents/flow diverters [n (per annum)] & \multicolumn{2}{|l|}{934} & \multicolumn{2}{|c|}{$3,925(785)$} & \\
\hline Coiling: 6 or more coils [n (per annum)] & 875 & & $4,254(\varepsilon$ & & \\
\hline Coiling: 2 or more coils [n (per annum)] & 763 & & 4,812 & & \\
\hline Clipping [n (per annum)] & 770 & & $5,394($ & & \\
\hline Diagnoses & & & & & \\
\hline Diagnosis (ICD10) & $\mathrm{n}$ & $\%$ & $n$ & $\%$ & \\
\hline aSAH from ICA (I60.0) & 186 & 6.91 & 926 & 6.86 & \\
\hline aSAH from MCA (I60.1) & 132 & 4.9 & 688 & 5.1 & \\
\hline aSAH from AComA (160.2) & 316 & 11.74 & 1,440 & 10.67 & \\
\hline aSAH from the PComA (160.3) & 25 & 0.93 & 89 & 0.66 & \\
\hline aSAH from BA (160.4) & 54 & 2.01 & 237 & 1.76 & \\
\hline aSAH from other intracranial arteries (I60.6) & 46 & 1.71 & 249 & 1.84 & \\
\hline Brain aneurysm, unruptured (I67.1) & 1,811 & 67.27 & 9,002 & 66.69 & \\
\hline aSAH, unspecified (I69.0) & 59 & 2.19 & 377 & 2.79 & \\
\hline Other malformations & 63 & 2.34 & 491 & 3.64 & \\
\hline Mortality rate & & & & & \\
\hline In-house deaths [n (per annum)] & 328 & & 1,400 & & \\
\hline Mortality rate [\%] & 9.65 & & 7.61 & & $p=0.0001^{*}$ \\
\hline
\end{tabular}

*"N-1"Chi-squared test; **Poisson's regression; AComA — anterior communicating artery; aSAH — aneurysmatic subarachnoid haemorrhage; BA — basilar artery; ICA — internal carotid artery; MCA — middle carotid artery; PComA — posterior communicating artery 
Conflict of interest: None.

Funding: None.

\section{References}

1. Jean WC, Ironside NT, Sack KD, et al. The impact of COVID-19 on neurosurgeons and the strategy for triaging non-emergent operations: a global neurosurgery study. Acta Neurochir (Wien). 2020; 162(6): 1229-1240, doi: 10.1007/s00701-020-04342-5, indexed in Pubmed: 32314059.

2. Chang De, Xu H, Rebaza A, et al. Protecting health-care workers from subclinical coronavirus infection. Lancet Respir Med. 2020; 8(3): e13, doi: 10.1016/s2213-2600(20)30066-7.

3. Sehba FA, Hou J, Pluta RM, et al. The importance of early brain injury after subarachnoid hemorrhage. Prog Neurobiol. 2012; 97(1): 14-37, doi: 10.1016/j.pneurobio.2012.02.003, indexed in Pubmed: 22414893.

4. Ministerstwo Zdrowia. Raport zakażeń koronawirusem (SARS-CoV-2). Koronawirus: informacje i zalecenia. https://www.gov.pl/web/koronawirus/wykaz-zarazen-koronawirusem-sars-cov-2.

5. Lindgren A, Vergouwen MDI, van der Schaaf I, et al. Endovascular coiling versus neurosurgical clipping for people with aneurysmal subarachnoid haemorrhage. Cochrane Database Syst Rev. 2018; 8(8): CD003085, doi: 10.1002/14651858.CD003085.pub3, indexed in Pubmed: 30110521.

6. Jiang Z, Chen Y, Zeng C, et al. Neurosurgical clipping versus endovascular coiling for patients with intracranial aneurysms: a systematic review and meta-analysis. World Neurosurg. 2020; 138: e191-e222, doi: 10.1016/j.wneu.2020.02.091, indexed in Pubmed: 32105881.

7. Drenck N, Grundtvig J, Christensen T, et al. Stroke admissions and revascularization treatments in Denmark during COVID-19. Acta Neurol Scand. 2021 [Epub ahead of print], doi: 10.1111/ane.13535, indexed in Pubmed: 34605006.
8. Mathiesen T, Arraez M, Asser T, et al. EANS Ethico-legal committee. A snapshot of European neurosurgery December 2019 vs. March 2020: just before and during the Covid-19 pandemic. Acta Neurochir (Wien). 2020; 162(9): 2221-2233, doi: 10.1007/s00701-02004482-8, indexed in Pubmed: 32642834.

9. Hulsbergen AFC, Eijkholt MM, Balak N, et al. Ethical triage during the COVID-19 pandemic: a toolkit for neurosurgical resource allocation. Acta Neurochir (Wien). 2020; 162(7): 1485-1490, doi: 10.1007/ s00701-020-04375-w, indexed in Pubmed: 32405671.

10. Fiorindi A, Vezzoli M, Doglietto F, et al. Aneurismal subarachnoid hemorrhage during the COVID-19 outbreak in a Hub and Spoke system: observational multicenter cohort study in Lombardy, Italy. Acta Neurochir (Wien). 2021 [Epub ahead of print], doi: 10.1007/s00701021-05013-9, indexed in Pubmed: 34694465.

11. Ganau M, Netuka D, Broekman M, et al. EANS IM Committee. Neurosurgeons and the fight with COVID-19: a position statement from the EANS Individual Membership Committee. Acta Neurochir (Wien). 2020; 162(8): 1777-1782, doi: 10.1007/s00701-020-04360-3, indexed in Pubmed: 32472377.

12. Yanez ND, Weiss NS, Romand JA, et al. CoVID-19 mortality risk for older men and women. BMC Public Health. 2020; 20(1): 1742, doi: 10.1186/s12889-020-09826-8, indexed in Pubmed: 33213391.

13. Renowden S, Nelson R. Management of incidental unruptured intracranial aneurysms. Pract Neurol. 2020; 20(5): 347-355, doi: 10.1136/practneurol-2020-002521, indexed in Pubmed: 32893193.

14. Tykocki T, Kostyra K, Czyż M, et al. Four-year trends in the treatment of cerebral aneurysms in Poland in 2009-2012. Acta Neurochir (Wien). 2014; 156(5): 861-868, doi: 10.1007/s00701-014-2006-z, indexed in Pubmed: 24499992.

15. Sweid A, Hammoud B, Bekelis $K$, et al. Cerebral ischemic and hemorrhagic complications of coronavirus disease 2019. Int J Stroke. 2020; 15(7): 733-742, doi: 10.1177/1747493020937189, indexed in Pubmed: 32501751. 